\title{
On the pulse-width statistics in radio pulsars
}

\author{
M. Kolonko ${ }^{1,2}$, J. Gil ${ }^{3}$, and K. Maciesiak ${ }^{3}$ \\ 1 Institute of Nuclear Physics, Radzikowskiego 152, 31-342 Kraków, Poland \\ 2 Institute of Astronomy, Jagiellonian University, Orla 171, 30-244 Kraków, Poland \\ 3 Institute of Astronomy, University of Zielona Góra, Lubuska 2, 65-265 Zielona Góra, Poland \\ e-mail: jag@astro.ia.uz.zgora.pl
}

Received 25 September 2003 / Accepted 28 July 2004

\begin{abstract}
We perform Monte Carlo simulations of pulsar periods, pulse-widths and magnetic inclination angles. Using the available observational data sets we study possible parent distribution functions by means of the Kolmogorov-Smirnov significance tests. We also use an additional condition that the numbers of generated interpulses, whether from both magnetic poles or from a single pole, are at the observed levels. We derived a model distribution function of magnetic inclination angles which has a local maximum near $\alpha=25^{\circ}$ and another weaker one near $\alpha=90^{\circ}$. The gamma function is a plausible distribution function describing pulsar periods. The average beaming fraction describing the fraction of observable radio pulsars is about 0.12 .
\end{abstract}

Key words. stars: pulsars: general - stars: neutron - stars: rotation

\section{Introduction}

Statistical studies of the pulse-width in mean profiles of radio pulsars are an important tool for investigations of the geometry of pulsar radiation. One especially important parameter that can be derived from such studies is the inclination angle between the magnetic and the spin pulsar axes. Early studies were carried out by Henry \& Paik (1969), Roberts \& Sturrock (1972, 1973), Backer (1976) and Manchester \& Lyne (1977). Since the amount of the available data was small, these papers suffered from problems of small number statistics. A more complete work was performed by Prószyński (1979) and Lyne \& Manchester (1988), who analyzed samples of about 200 pulse-width data measured near $400 \mathrm{MHz}$. Although the database used in these papers was quite rich, the pulse-width measurements were contaminated by the interstellar scattering dominating at low radio frequencies. More recently Gil \& Han (1996, GH96 hereafter) compiled a new database of 242 pulsewidths $W_{10}$ (corresponding to about $10 \%$ of the maximum intensity) measured at a higher radio frequency (near $1.4 \mathrm{GHz}$ ), which was relatively unbiased compared to the lower frequency data. GH96 used their pulse-width database to perform Monte Carlo simulations in an attempt to derive the distribution statistics of pulsar periods, pulse widths, magnetic inclination angles and rates of interpulses. They concluded by comparing the simulated and observed (or observationally derived) quantities that the observed distribution of the inclination angles resembles a sine function following from the flat (random) distribution in the parent population, and that the probability (beaming fraction) of observing a pulsar was about 0.16 . GH96 also pointed out that the rates of interpulse occurrence should be considered as an important aspect of pulsar population studies.

On the other hand, Tauris \& Manchester (1998, henceforth TM98) using a different method based on an analysis of the indirectly derived polarization position angles and magnetic inclination angles concluded that the observed distribution of the latter is cosine-like rather than sine-like as suggested by GH96. They also obtained the beaming factor $0.1 \pm 0.02$, considerably lower than 0.16 obtained by GH96. TM98 pointed out a likely source of this discrepancy, namely the incorrect assumption used by GH96 that the observed distribution and the parent distribution of pulsar periods are similar. Recently, Zhang et al. (2003, henceforth ZJM03) followed the Monte-Carlo simulation scheme developed by GH96. ZJM03 argued that both the parent distribution function and the observed distribution of pulsar periods can be modelled by the gamma function but with different values of the free parameters, and their Monte-Carlo simulations included searching for a 2D grid of these parameters. As a result, ZJM03 concluded that indeed the cosinelike distribution (suggested by TM98) is much more suitable to model the inclination angles in the parent pulsar population than the flat distribution (suggested by GH96). They argued that the most plausible parent distribution is a modified cosine function, which has a peak around $25^{\circ}$ and another weaker peak near $90^{\circ}$. They also obtained the beaming factor $\sim 0.12$, consistent with the result of TM98.

As emphasized by ZJM03 in the conclusions of their paper, neither they nor TM98 considered potentially important constraints related to the observable interpulse emission. Although GH96 did consider the issue of interpulse emission, their estimate of rates of occurrence was not quite correct (see Sect. 3.4 
in this paper). Moreover, their statistical analysis was biased by the incorrect assumption mentioned above concerning the parent distribution of pulsar period. In this work we follow the simulation scheme of ZJM03 but we include the actual rates of interpulse occurrences. We demonstrate that a pure cosine distribution of the magnetic inclination angles generates too few interpulses compared to observations and should be rejected as a plausible distribution function. We found that the modified cosine function of ZJM03 (see Eq. (9)) is much better in this respect, mainly because of the weak second maximum near $\alpha=90^{\circ}$. Both the above distribution functions can reproduce the observed distributions of pulse-widths, pulsar periods and inclination angles almost equally well, and thus the observed interpulse statistics provide the most restrictive constraint discriminating between different trial distribution functions.

\section{Basic formulae}

\subsection{Pulse-widths}

It is generally accepted that the pulsar radio emission is relativistically beamed (Graham-Smith 2003) along the open dipolar field lines (to within $1 / \gamma$, where $\gamma \sim 100$ is the Lorentz factor of the emitting sources). Thus, the pulse width $W_{10}$ at the level of $10 \%$ of the maximum profile intensity can be written as

$W_{10}=4 \arcsin \left\{\frac{\sin \left[\left(\rho_{10}+\beta\right) / 2\right] \sin \left[\left(\rho_{10}-\beta\right) / 2\right]}{\sin \alpha \cdot \sin (\alpha+\beta)}\right\}^{1 / 2}$

(Gil 1981), where $\rho_{10}$ is the beam-width corresponding to the $10 \%$ intensity level, $\alpha$ is the inclination angle of the magnetic axis to the spin axis, $\beta$ is the impact angle of the closest approach of the line-of-sight to the magnetic axis, and thus $\xi=\alpha+\beta$ is the observer angle between the spin axis and the line-of-sight. It is worth noting that Eq. (1) assumes the symmetry of a pulsar beam with respect to the fiducial plane containing both the spin and the magnetic pulsar axes. Thus, the appropriate pulse width database should contain only pulsars with symmetrical profiles for which $W=2 \varphi$, where $\varphi$ is the pulse longitude measured from the fiducial phase corresponding to the fiducial plane (e.g. GH96).

\subsection{Opening angles}

The opening angle of the radio beam (beam-width) corresponding to the $10 \%$ intensity level can be derived from pulse width measurements $W_{10}$ (and $\alpha, \beta$ values) in the form of the socalled $\rho-P$ relation (Gil et al. 1984). Lyne \& Manchester (1988) obtained $\rho_{10} \approx 6.5 P^{-1 / 3}$ for the pulse-width data at $408 \mathrm{MHz}$, which scaled to $1.4 \mathrm{GHz}$ is

$\rho_{10} \approx 5.8 P^{-0.33}$.

However, Biggs (1990) reanalyzed the same data sample and concluded that

$\rho_{10} \approx 5.6 P^{-0.5}$.

Rankin (1993a,b) analyzed a large amount of available pulsewidth data interpolated to the frequency $\sim 1 \mathrm{GHz}$ and obtained a bimodal distribution of the opening angles, according to which for a given pulsar period the opening angle adopts one of the two preferred values. This result was later confirmed at frequency $\sim 1.4 \mathrm{GHz}$ by Gil et al. (1993, GKS93 henceforth) and independently by Kramer et al. (1994) and the resulting $\rho-P$ relation reads

$\rho_{10}=\left\{\begin{array}{l}6.3 P^{-0.5}, \\ 4.9 P^{-0.5},\end{array}\right.$

with smaller angles preferred at shorter periods. GKS93 argued that 4.9 is preferred in $80 \%$ of pulsars with $P<0.7 \mathrm{~s}$, and this constraint was used by GH96 (their Table 2), while ZJM03 used 4.9 for $P<0.7$ s and 6.3 for other periods (their Table 1 ). We denote the former option by Eq. (4a) and the latter one by Eq. (4b) in Table 2. It is worth noticing that $\rho-P$ relations expressed by Eqs. (2)-(4) are, to some extent, equivalent. In fact, Eq. (2) seems to reflect the tendency of smaller angles being preferred at short periods in Eq. (4), and Eq. (3) represents the average of both values appearing in Eq. (4). Nevertheless, we use all the above relations in our simulation procedure (Sect. 4).

\subsection{Pulsar axes}

It is reasonable to assume that both the rotation axis and the observer's direction are randomly oriented in space. Thus, the probability density function for the observer angle $\xi=\alpha+\beta$ is

$f(\xi)=\sin (\alpha+\beta)$

However, the distribution of the inclination angle may depend on many unknown factors, and the most common probability density functions in the parent population of pulsars include the flat function

$f(\alpha)=\frac{2}{\pi}$

for the random distribution, the sine function

$f(\alpha)=\sin \alpha$

for the sine-like distribution, and the cosine function

$f(\alpha)=\cos \alpha$

for the cosine-like distribution. One can consider some more complicated probability density functions. For example, ZJM03 argued that the plausible parent distribution of the inclination angle can be described in the form

$f(\alpha)=\frac{0.6}{\cosh (3.5(\alpha-0.43))}+\frac{0.15}{\cosh (4.0(\alpha-1.6))}$.

This function has a weak local maximum near $\alpha=\pi / 2$, which is an important feature with regard to the interpulse occurrence statistics. We test all the above functions in our Monte Carlo simulation procedure (Sect. 4). 
Table 1. Results of Monte Carlo simulations - reproduction of Table 1 in ZJM03.

\begin{tabular}{|c|c|c|c|c|c|c|c|c|c|c|c|c|}
\hline \multirow{3}{*}{$\begin{array}{c}\rho-P \\
\text { relation }^{\dagger} \\
\rho_{10}(P)\end{array}$} & \multirow{3}{*}{$\begin{array}{c}\begin{array}{c}\text { Inclination } \\
\text { angle }\end{array} \\
f(\alpha)\end{array}$} & \multirow{2}{*}{\multicolumn{2}{|c|}{$\begin{array}{c}\begin{array}{c}\text { Pulsar } \\
\text { period }\end{array} \\
f(P)^{\dagger \dagger}\end{array}$}} & \multicolumn{6}{|c|}{$\begin{array}{c}\text { Kolmogorov-Smirnov } \\
\text { statistics }^{\dagger \dagger}\end{array}$} & \multirow{3}{*}{$\begin{array}{c}\begin{array}{c}\text { Beaming } \\
\text { fraction }\end{array} \\
\mathrm{f}\end{array}$} & \multicolumn{2}{|c|}{$\begin{array}{l}\text { Interpulse } \\
\text { rates }\end{array}$} \\
\hline & & & & \multicolumn{2}{|c|}{$P$} & \multicolumn{2}{|c|}{$W_{10}$} & \multicolumn{2}{|c|}{$\alpha$} & & \multirow[b]{2}{*}{ DP-IP } & \multirow[b]{2}{*}{ SP-IP } \\
\hline & & $m$ & $a$ & $\mathcal{D}$ & $\mathcal{P}$ & $\mathcal{D}$ & $\mathcal{P}$ & $\mathcal{D}$ & $\mathcal{P}$ & & & \\
\hline A. & 1. $\cos \alpha$ & 0.38 & 2.21 & 0.049 & 0.017 & 1.068 & 0.236 & 0.139 & 0.007 & 0.119 & $0.25 \%$ & $0.62 \%$ \\
\hline A. & 2. Eq. (9) & 0.38 & 2.204 & 0.046 & 0.029 & 0.108 & 0.009 & 0.102 & 0.093 & 0.123 & $2.24 \%$ & $0.36 \%$ \\
\hline A. & 3. $2 / \pi$ & 0.278 & 2.277 & 0.151 & $10^{-18}$ & 0.048 & 0.666 & 0.354 & $10^{-13}$ & 0.162 & $3.58 \%$ & $0.39 \%$ \\
\hline A. & 4. $2 / \pi$ & 0.411 & 2.101 & 0.046 & 0.028 & 0.105 & 0.012 & 0.360 & $10^{-14}$ & 0.147 & $3.37 \%$ & $0.41 \%$ \\
\hline B. & 1. $\cos \alpha$ & 0.39 & 2.339 & 0.054 & 0.007 & 0.121 & 0.002 & 0.140 & 0.006 & 0.121 & $0.28 \%$ & $1.01 \%$ \\
\hline B. & 2. Eq. (9) & 0.4 & 2.29 & 0.051 & 0.011 & 0.157 & $10^{-5}$ & 0.1 & 0.110 & 0.126 & $2.58 \%$ & $0.69 \%$ \\
\hline B. & 3. $2 / \pi$ & 0.278 & 2.277 & 0.184 & 0.007 & 0.148 & $10^{-4}$ & 0.347 & $10^{-13}$ & 0.179 & $4.71 \%$ & $0.75 \%$ \\
\hline B. & 4. $2 / \pi$ & 0.4 & 2.255 & 0.049 & 0.017 & 0.101 & 0.018 & 0.354 & $10^{-14}$ & 0.154 & $3.72 \%$ & $0.49 \%$ \\
\hline C. & 1. $\cos \alpha$ & 0.38 & 2.27 & 0.054 & 0.006 & 0.086 & 0.066 & 0.137 & 0.008 & 0.122 & $0.29 \%$ & $0.90 \%$ \\
\hline C. & 2. Eq. (9) & 0.37 & 2.27 & 0.057 & 0.009 & 0.137 & 0.019 & 0.1 & 0.107 & 0.128 & $2.54 \%$ & $0.51 \%$ \\
\hline C. & 4. $2 / \pi$ & 0.378 & 2.262 & 0.047 & 0.023 & 0.085 & 0.067 & 0.355 & $10^{-14}$ & 0.153 & $3.57 \%$ & $0.43 \%$ \\
\hline
\end{tabular}

${ }^{\dagger} \rho-P$ relation (beam radius): $\mathrm{A}-\rho_{10}=5.8 P^{-1 / 3}$ (Eq. (2)), $\mathrm{B}-\rho_{10}=5.6 P^{-1 / 2}$ (Eq. (3)),

$\mathrm{C}-\rho_{10}=4.9 P^{-1 / 2}$ for $P<0.7 \mathrm{~s}$ and $\rho_{10}=6.3 P^{-1 / 2}$ for others (Eq. $\left.(4 \mathrm{~b})\right)$

†† gamma function $f(P)=G_{0}(P / m)^{a-1} e^{-(P / m)}$ (Eq. (10))

$\dagger^{\dagger \dagger} \mathrm{K}-\mathrm{S}$ tests: $\mathcal{D}$ - maximum difference between cumulative distribution functions corresponding to the observed and the simulated data samples, $\mathcal{P}$ - probability that both the observed and the simulated data sets are drawn from the same parent distribution.

Table 2. Results of Monte Carlo simulations.

\begin{tabular}{|c|c|c|c|c|c|c|c|c|c|c|c|c|c|}
\hline \multirow[t]{3}{*}{ No } & \multirow{3}{*}{$\begin{array}{c}\rho-\mathrm{P} \\
\text { relation }\end{array}$} & \multirow{3}{*}{$\begin{array}{c}\begin{array}{c}\text { Inclination } \\
\text { angle }\end{array} \\
f(\alpha)\end{array}$} & \multirow{3}{*}{\multicolumn{2}{|c|}{$\begin{array}{l}\text { Pulsar } \\
\text { period }\end{array}$}} & \multicolumn{6}{|c|}{$\begin{array}{c}\text { Kolmogorov-Smirnov } \\
\text { statistics }^{\dagger}\end{array}$} & \multirow{3}{*}{$\begin{array}{c}\begin{array}{c}\text { Beaming } \\
\text { fraction }\end{array} \\
\qquad f\end{array}$} & \multicolumn{2}{|c|}{$\begin{array}{l}\text { Interpulse } \\
\text { rates }\end{array}$} \\
\hline & & & & & \multicolumn{2}{|c|}{$P$} & \multicolumn{2}{|c|}{$W_{10}$} & \multicolumn{2}{|c|}{$\alpha$} & & \multirow[b]{2}{*}{ DP-IP } & \multirow[b]{2}{*}{ SP-IP } \\
\hline & & & & & $\mathcal{D}$ & $\mathcal{P}$ & $\mathcal{D}$ & $\mathcal{P}$ & $\mathcal{D}$ & $\mathcal{P}$ & & & \\
\hline \multirow[t]{2}{*}{1.} & Eq. (4a) & Eq. (9) & & & & & & & & & & & \\
\hline & & & $m=0.34$ & $a=2.52$ & 0.054 & 0.006 & 0.124 & 0.002 & 0.093 & 0.156 & 0.128 & $2.07 \%$ & $0.74 \%$ \\
\hline \multirow[t]{2}{*}{2.} & Eq. (3) & Eq. (9) & & & & & & & & & & & \\
\hline & & & $m=0.36$ & $a=2.6$ & 0.055 & 0.006 & 0.127 & 0.001 & 0.101 & 0.103 & 0.121 & $2.55 \%$ & $0.64 \%$ \\
\hline 3. & & & $m=0.32$ & $a=2.6$ & 0.054 & 0.006 & 0.117 & 0.004 & 0.098 & 0.120 & 0.125 & $2.44 \%$ & $0.53 \%$ \\
\hline \multirow[t]{2}{*}{4.} & Eq. (2) & Eq. (9) & & & & & & & & & & & \\
\hline & & & $x_{0}=0.6$ & $a_{0}=0.42$ & 0.053 & 0.008 & 0.090 & 0.049 & 0.107 & 0.072 & 0.123 & $2.38 \%$ & $0.57 \%$ \\
\hline \multirow[t]{2}{*}{5.} & Eq. (4b) & Eq. (9) & & & & & & & & & & & \\
\hline & & & $x_{0}=0.64$ & $a_{0}=0.38$ & 0.058 & 0.003 & 0.115 & 0.004 & 0.109 & 0.061 & 0.126 & $2.25 \%$ & $0.51 \%$ \\
\hline
\end{tabular}

† see Table 1 for explanation.

\subsection{Pulsar periods}

GH96 demonstrated that the observed distribution of periods of 516 pulsars with $4.2 \mathrm{~s}>P>0.05 \mathrm{~s}$ (Pulsar Catalog Taylor et al. 1993) can be well fitted by the gamma function

$f(P)=G_{0} x^{a-1} \mathrm{e}^{-x}$

with $x=P / m$, where $m=0.3$ and $a=2.5\left(G_{0}\right.$ is the normalization constant dependent on $a$ ). ZJM03 used this function to fit the period distribution in a much larger sample of 1164 pulsar periods $4.2 \mathrm{~s}>P>0.05 \mathrm{~s}$ and obtained $m=0.278$ and $a=2.277$. Although the parent distribution of periods is certainly different from the observed distribution, it is convenient to represent the former also in the general form of a gamma function (Eq. (10)), with values of $m$ and $a$ treated as free model parameters that can be derived in 2D grid search implemented in the Monte Carlo simulations (Sect. 4).
For a comparison one can also try some other trial probability density distribution functions, like the Lorentzian distribution function

$f(P)=\frac{L_{0}}{1+\left(P-x_{0}\right)^{2} / a_{0}^{2}}$,

or even the Gaussian distribution function

$f(P)=\frac{1}{\sqrt{2 \pi} \sigma_{0}} \exp \left[\frac{-\left(P-x_{0}\right)^{2}}{2 \sigma_{0}}\right]$.

Again, the values of free parameters can be derived in a 2D grid search within the Monte Carlo simulations (Sect. 4).

\subsection{Detection conditions}

Following the arguments given by Lyne \& Manchester (1988), GH96 and Mitra \& Deshpande (1999) we first assume that the 
pulsar beam is circular or almost circular. If this is the case, then the detection condition (see GH96 for details) is:

$\rho>|\beta|$

for the typical Main Pulse (MP) emission. However, one should also include the rare interpulse (IP) emission, seen in a few percent of pulsars. Two models were proposed to explain this emission occurring about 180 degrees of longitude from the centroid of the MP: the double pole (DP-IP) model, in which MP and IP originate from opposite magnetic poles (Rickett \& Lyne 1968; Manchester \& Lyne 1977), and the single pole (SP-IP), in which both MP and IP are associated with the same pole. The detection condition for the DP-IP model is

$\rho>\pi-2 \alpha-\beta$

(e.g. GH96), which favors a nearly orthogonal rotators $(\alpha \sim \pi / 2)$. Both MP and IP in pulsars that belong to the DP-IP class show relatively narrow duty cycles, contrary to the broad profiles in the SP-IP class associated with nearly aligned rotators $(\alpha \sim 0)$. There are two possibilities within the SP-IP: in the first one the MP and the IP represent two cuts through one conical beam (Manchester 1978; Manchester \& Lyne 1977). The other possibility is when the line-of-sight stays in the overall pulsar beam for the entire pulsar period, so the MP and IP correspond to cuts through two nested conical beams (Gil 1983). The detection conditions for these two versions of SP-IP models are

$\rho>\sqrt{2 \alpha^{2}+\beta^{2}}$

and

$\rho>2 \alpha+\beta$

respectively. Both these conditions are checked alternatively. One should mention that only the latter condition was used by GH96, which resulted in too low rates of SP-IP compared to observations.

One can also consider a quite natural possibility that the pulsar beam has a tendency towards meridional compression, with the ratio of minor to major ellipse axes

$R \approx \cos \frac{\alpha}{3} \sqrt{\cos \left(\frac{2}{3} \alpha\right)}$

depending on the inclination angle (Biggs 1990; McKinnon 1993). Then the appropriate detection conditions (generalizations of Eqs. (13)-(15)) are:

$|\beta|<\sqrt{R^{2} \rho^{2}+\beta^{2}\left(1-R^{2}\right)}$

for the Main Pulse emission,

$r R>\pi-2 \alpha-\beta$

for the DP-IP emission, and

$r R>2 \alpha+\beta$ or $r R>\sqrt{2 \alpha^{2}+\beta^{2}}$ for the SP-IP emission, where

$r=\sqrt{\rho^{2}+\beta^{2}\left(\frac{1}{R^{2}}-1\right)}$

is the latitudinal beam dimension. Note that $R \leq 1$ and $r \geq \rho$, where $\rho$ is the observationally deduced beam radius (see GH96 for details).

\section{Observational data}

In our statistical analysis we compare the simulated distributions with the directly ( $W_{10}, P$, IP occurrences) or indirectly $(\alpha)$ observed data by means of the Kolmogorov-Smirnov (K-S henceforth) significance tests. Using the numerical methods given by Press et al. (1992) we compute the maximum distance $\mathcal{D}$ (maximum difference between cumulative distribution functions corresponding to the observed and simulated data sets) as well as the significance $\mathcal{P}$ of any non zero value of $\mathcal{D}$. The value of $\mathcal{P}$ represents the probability that both the simulated and the observed data sets are drawn from the same parent distribution. We adopt an arbitrary criterion that $\mathcal{P}>0.001(0.1 \%)$ for distributions of $P, W_{10}$ and $\alpha$ at the same time.

\subsection{Pulsar periods}

Using the available database of pulsar parameters ${ }^{1}$ we selected a sample of 1165 normal pulsars with periods $P$ with $0.02 \mathrm{~s}<$ $P<8.52$ s (Fig. 1). We rejected all millisecond and other recycled pulsars, since they represent different period and magnetic inclination angle populations than typical pulsars. This sample of observed pulsar periods can be fitted by the gamma function expressed by Eq. (10) with $m=0.28$ and $a=2.28$ (see upper panel in Fig. 1).

\subsection{Pulse-widths}

We use the database of 238 pulse-width measurements $W_{10}$ taken at the frequency of about $1.4 \mathrm{GHz}$ (upper panel in Fig. 2), compiled by GH96 (their Table 1 and Fig. 1). These measurements were carefully selected from the available databases to satisfy all criteria imposed by the symmetry of Eq. (1), (see comments below this equation and GH96 for more details). ZJM03 demonstrated that another available database (containing 265 pulsars), that of Gould \& Lyne (1998), is equivalent to that of GH96 in the sense that the values of $W_{10}$ are roughly the same in both these databases. It is worth noting that these two databases are based on completely different profile widths measurements.

\subsection{Inclination angles}

While the values of $W_{10}$ and $P$ as well as the interpulse occurrences are direct observational quantities, the values of the inclination angles $\alpha$ can only be indirectly observed by means

\footnotetext{
1 http://www.atnf.csiro.au/research/pulsar/psrcat
} 


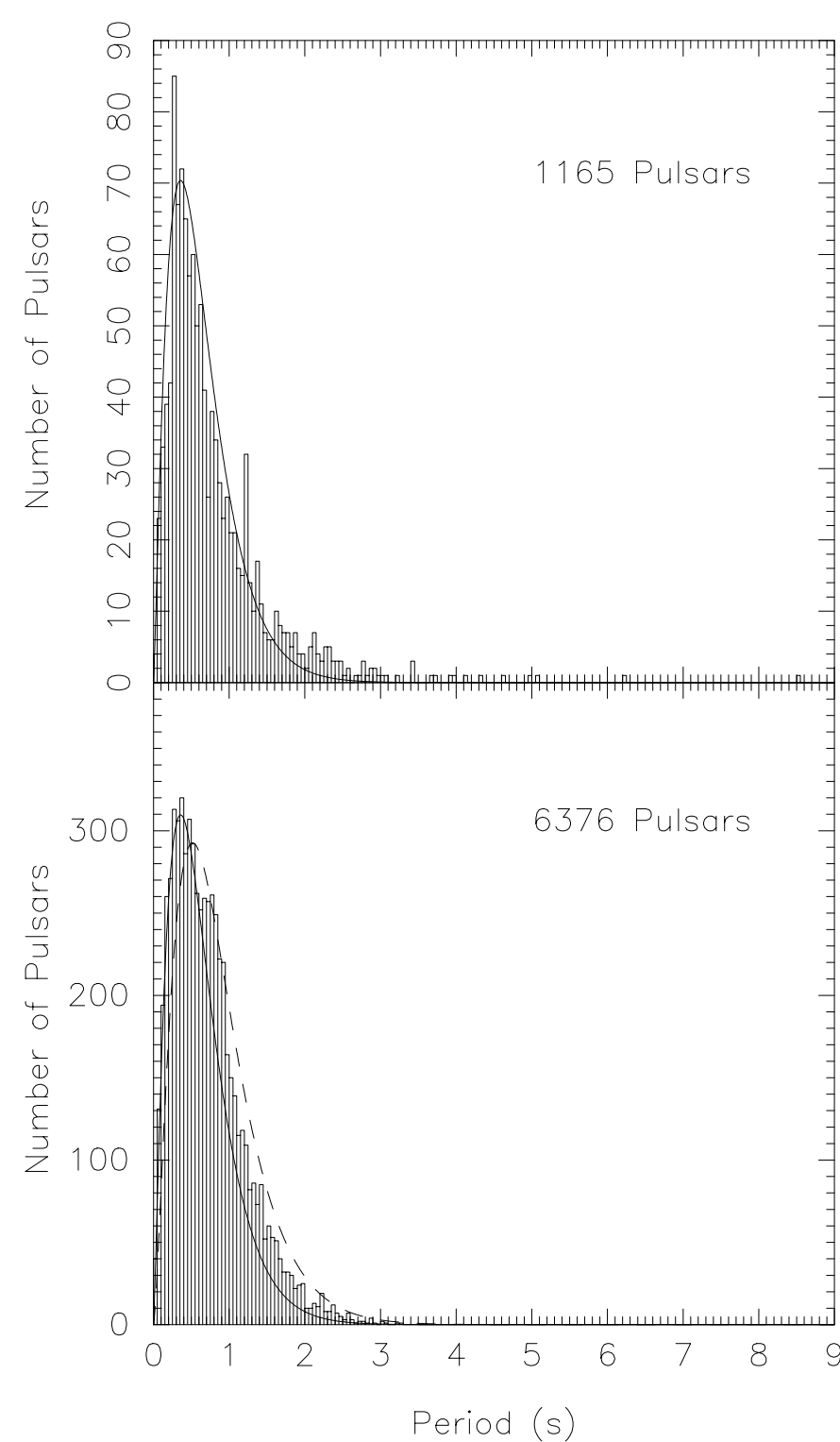

Fig. 1. Observed distribution of 1165 pulsar periods with $0.02 \mathrm{~s}<$ $P<8.52$ s (upper panel) and simulated distribution corresponding to case (1) in Table 2 (lower panel). The solid lines represent the analytical fit to the observed distribution in the form of a gamma function with $m=0.28$ and $a=2.28$, while the dashed line represents the parent density distribution gamma function with $m=0.34$ and $a=2.52$.

of the polarization measurements (Lyne \& Manchester 1988; Rankin 1990, 1993a,b; Gould 1994). Following the arguments of ZJM03 we used the database of the inclination angles compiled by Rankin (1993a,b). This database contains 149 measurements of the magnetic inclination angles, whose distribution is presented in the upper panel of Fig. 3. Most of these values were derived not from the polarization measurements but from the so-called pulse-width/period relation established for core components by Rankin (1993a). However, as demonstrated by TM98, values of inclination angles derived by this method are roughly consistent with those estimated from the polarization measurements (LM88; Gould 1994).

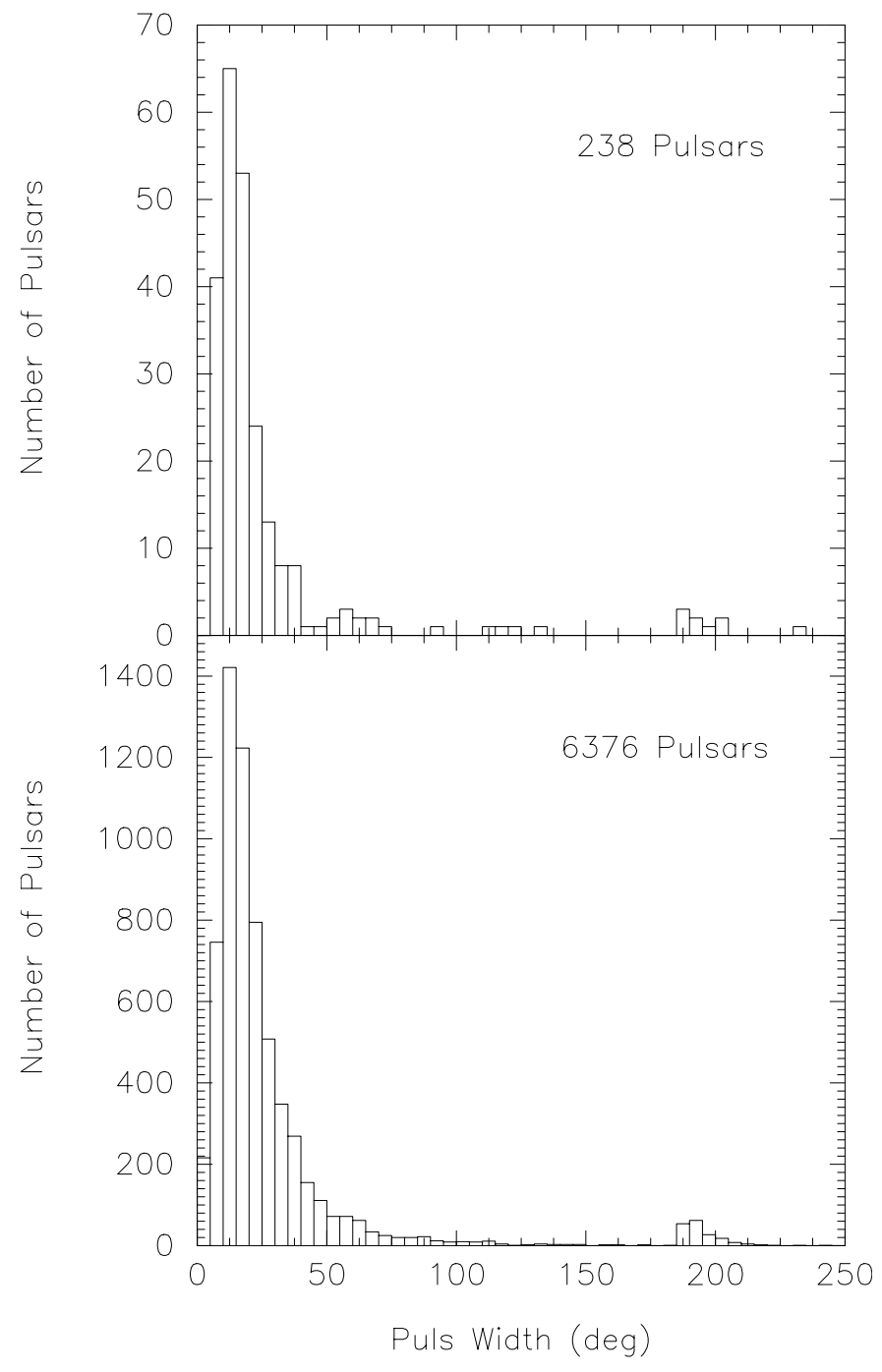

Fig. 2. Observed distribution of 238 pulse widths $W_{10}$ taken from GH96 (upper panel) and simulated distribution corresponding to case (1) in Table 2 (lower panel).

\subsection{Interpulse emission}

The fraction of interpulses in the observed sample of pulsars provides useful information about pulsar geometry (see GH96). However, the statistical studies of this phenomenon are difficult since the ratio of amplitudes of the interpulse to the mainpulse is often about $1 \%$ and varies with frequency (Hankins $\&$ Fowler 1986). The only representative sample of interpulses can be found in Table 6 of the Catalog of 558 pulsars (Taylor et al. 1993). There are 22 pulsars with interpulses $(\sim 4 \%)$ in this sample, with only 3 certain cases (B0826-34, B0950+08, B1929+10) corresponding to a single magnetic pole (Lyne \& Manchester 1988, their Fig. 8). Most likely PSR B1848+04 with a very broad main-pulse also belongs to this category. To be consistent with our selection of pulsar periods (Sect. 3.2) one should exclude millisecond and other recycled pulsars from both the total pulsar sample and from the sample of pulsars with interpulses (this was not done in GH96, who incorrectly estimated rates of interpulse occurrences as a result of this mistake). Table 3 lists our final sample of interpulse 


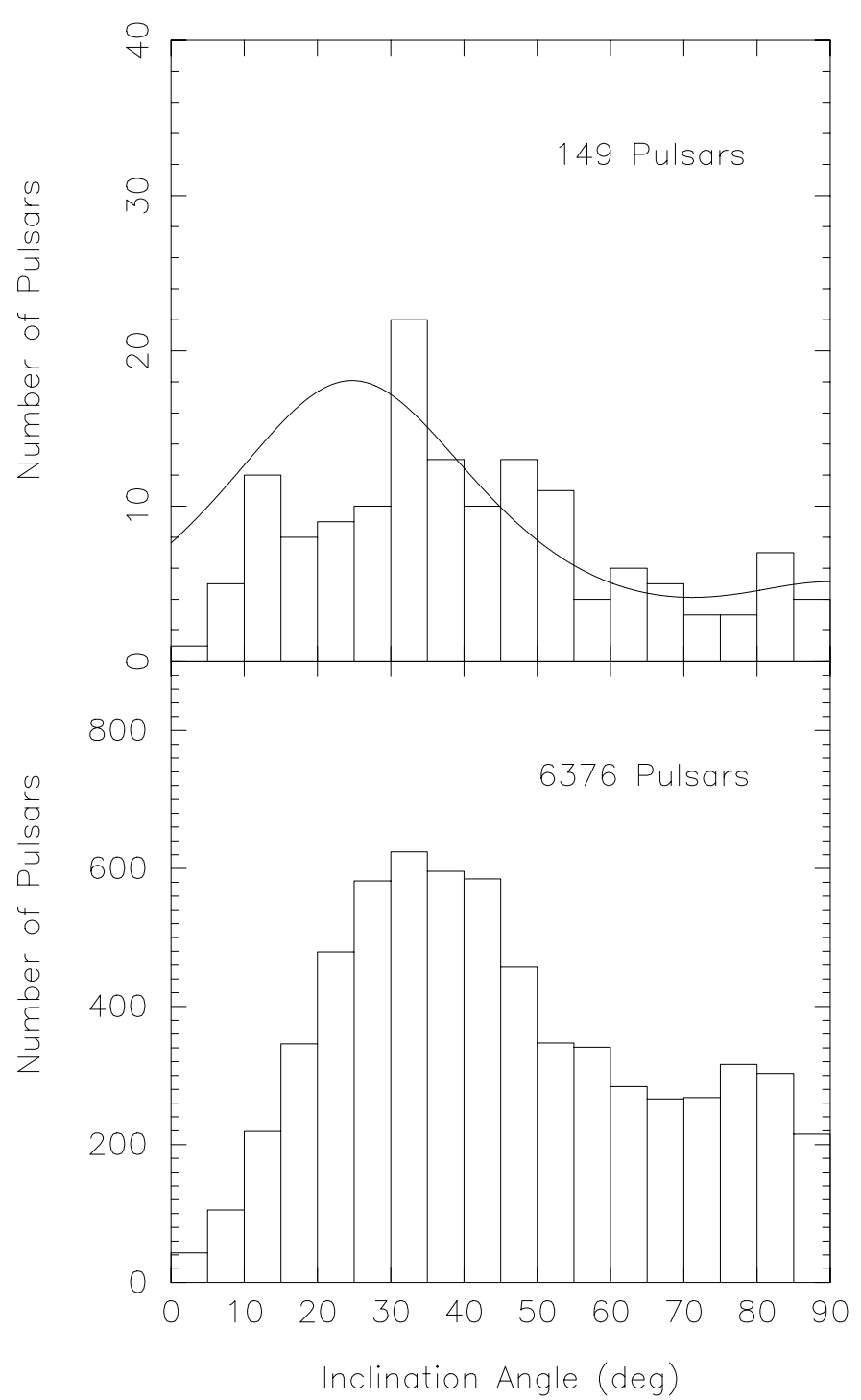

Fig. 3. Observed distribution of 149 magnetic inclination angles $\alpha$ taken from Rankin (1993b) (upper panel) and simulated distribution corresponding to case (1) in Table 2 (lower panel). The solid line represents the parent density distribution function expressed by Eq. (9).

pulsars, in which we identified 10 DP-IP and 4 SP-IP cases $^{2}$. This corresponds to the rates of occurrence of above $2 \%$ and $0.8 \%$ for DP-IP and SP-IP, respectively. For a comparison, in the published sample of 420 pulsars found during the Parkes multibeam pulsar survey (Manchester et al. 2001; Morris et al. 2002; Kramer et al. 1994) one can clearly identify 4 cases of

\footnotetext{
${ }^{2}$ In most cases the classification is obvious, although PSR B1259-63 is a bit problematic. We classified this pulsar as DP-IP, while Manchester (1996) argued that it is just a case of a wide hollow-cone. However, we note that interpulse properties of this pulsar are almost identical to those of the Crab pulsar, which is commonly classified as an almost orthogonal rotator with an interpulse corresponding to the opposite magnetic pole. In both these pulsars the separation $\left(145^{\circ}\right)$ between the MP and IP is frequency independent, which seems to contradict the wide hollow-cone hypothesis. We note that the classification uncertainty of this pulsar does not significantly change the observed percentages of interpulse emission in normal pulsars (Table 3).
}

DP-IP and 1 case of SP-IP, and a few low intensity candidates. This gives lower limits of about $1 \%$ and $0.24 \%$, respectively. It is difficult to estimate the actual rates of IP occurrence until a sensitive search for interpulses in the newly discovered pulsars is made. We assume that the plausible distributions of periods $P$ and inclination angles $\alpha$ in the parent pulsar population should be able to explain about $2 \%$ of DP-IP and slightly above $0.5 \%$ of SP-IP in the normal pulsar population (excluding millisecond and other recycled pulsars).

\section{Monte Carlo simulations}

We performed Monte Carlo simulations of the pulse widths $W_{10}$, pulsar periods $P$ and inclination angles $\alpha$, in an attempt to reproduce the observed distributions of these quantities. We have used the random number generator given in Press et al. (1992). Our simulation procedure can be described as a number of sequential steps:

(1) Generate the inclination angle $\alpha$ as a random number with the parent probability density function $f(\alpha)$ corresponding to Eqs. (6)-(9), respectively.

(2) Generate the observer angle $\xi=\alpha+\beta$ as a random number with the parent probability density function $f(\xi)=\sin \xi$ (Eq. (5)), and calculate the impact angle $\beta=\xi-\alpha$.

(3) Generate the pulsar period $P$ as a random number with the parent probability density function $f(P)$ corresponding to Eqs. (10)-(12), respectively. Record the free model parameters of each function used.

(4) For a given value of $P$ calculate the opening angle $\rho_{10}$ corresponding to Eqs. (2)-(4), respectively.

(5) Check the detection condition corresponding to Eqs. (13)-(15) for $N_{\text {tot }}=50000$ simulated pulsars for each combination of $\rho-P$ relations (Eqs. (2)-(4)) and distribution functions $f(P)$ (Eqs. (10)-(12)) and $f(\alpha)$ (Eqs. (6)-(9)). Record the number of observed pulsars $N_{\text {obs }}$. Calculate the beaming fraction $f=N_{\text {obs }} / N_{\text {tot }}$, as well as the rates of detected interpulses from two magnetic poles (DP-IP) and from a single magnetic pole (SP-IP).

(6) For each set of parameters $\alpha, \beta$ and $\rho_{10}(P)$ corresponding to the observable pulsar calculate the pulse width $W_{10}$ according to Eq. (1), and record the relevant information $\left(W_{10}, P, \alpha, I P\right)$.

(7) Judge the statistical significance using the K-S tests for the simulated and observed distributions of pulse width $W_{10}$, period $P$ and inclination angle $\alpha$.

\subsection{Reproduction of previous results of GH96 and ZJM03}

To make sure that our simulation software works properly, we began with a reproduction of results given in the previous statistical works of GH96 and ZJM03. As expected, we managed to reproduce the K-S statistics for $W_{10}$, beaming fraction $f$ and the rates of observed interpulses given in Table 2 of GH96. However, the K-S test for pulsar periods (not included in GH96) resulted in very low probabilities (below $10^{-6}$ ), confirming the suggestion of TM98 that the distribution of periods 
Table 3. Interpulse emission in normal pulsars (after Table 6 in Taylor et al. 1993).

\begin{tabular}{cccccc}
\hline \hline No. & PSR & P (s) & $\begin{array}{c}\text { Fractional } \\
\text { amplitude }\end{array}$ & $\begin{array}{c}\text { Phase } \\
\text { separation }\left(^{\circ}\right)\end{array}$ & $\begin{array}{c}\text { How many } \\
\text { poles? }\end{array}$ \\
\hline 1. & B0531+21 & 0.033 & 0.6 & 145 & DP \\
2. & B0823+26 & 0.530 & 0.005 & 180 & DP \\
3. & B0826-34 & 1.848 & 0.1 & 180 & SP \\
4. & B0906-49 & 0.106 & 0.24 & 176 & DP \\
5. & B0950+08 & 0.253 & 0.012 & 210 & SP \\
6. & B1055-52 & 0.197 & 0.5 & 205 & DP \\
7. & B1259-63 & 0.047 & 0.75 & 145 & DP \\
8. & B1702-19 & 0.298 & 0.15 & 180 & DP \\
9. & B1719-37 & 0.236 & 0.15 & 180 & DP \\
10. & B1736-29 & 0.322 & 0.4 & 180 & DP \\
11. & B1822-09 & 0.768 & 0.05 & 185 & DP \\
12. & B1848+04 & 0.285 & 0.2 & 200 & SP \\
13. & B1929+10 & 0.226 & 0.018 & 170 & SP \\
14. & B1944+17 & 0.441 & 0.005 & 175 & DP \\
\hline
\end{tabular}

in the parent pulsar population is significantly different from that of the observed distribution. As we argue later in this paper, a plausible parent distribution of periods can be expressed as a gamma function (Eq. (10)) with $m=0.34 \pm 0.02$ and $a=2.52 \pm 0.04$, in contrast to $m=0.3$ and $a=2.5$ obtained for the observed sample of 516 pulsar periods by GH96, or $m=0.28$ and $a=2.28$ obtained for 1165 periods used in this paper (see also ZJM03).

The twelve entries in Table 1 correspond to cases A1-A4, B1-B4 and C1-C4 from Table 1 in ZJM03 (we excluded cases D1-D4 since for $s=5.8 / 8.8=0.65$ they are equivalent to cases A1-A4). As one can see, we reproduced quite well the results of K-S tests for $P, W_{10}$ and $\alpha$, as well as the values of the beaming fraction $f$. We added the rates of the interpulse occurrence and concluded that the pure $\cos \alpha$ distribution of the parent inclination angles (A1, B1 and C1) generates too few DP-IP (about $0.3 \%$ compared to about $2 \%$ observed). Thus, although the pure $\cos \alpha$ distribution gives very good results for K-S tests (consistent with the results of TM98 and ZJM03), it should be rejected on the grounds of unacceptable interpulse statistics. We found that the modified cosine distribution of ZJM03 (see Eq. (9)) not only gives the plausible results of K-S test, but also reproduces the rate of occurrence of both DP-IP $(\sim 2 \%)$ and SP-IP $(0.5 \%-0.7 \%)$.

\subsection{Other new results}

We have examined 19200 combinations of distribution functions of pulsar periods (Eqs. (10)-(12) with 200 combinations of parameters in each case), opening angles (Eqs. (2)-(4a,b)), inclination angles (Eqs. (6)-(9)) as well as two options of the beam shape: circular (Eqs. (13)-(16)) and elliptical (Eqs. (17)-(21)). We recorded only those cases in which the following conditions were simultaneously satisfied: the probabilities $\mathcal{P}$ that the observed and simulated distributions of $P, W_{10}$ and $\alpha$ exceeded 0.001 , as well as that the rates of occurrence of DP-IP and SP-IP exceeded $2 \%$ and $0.5 \%$, respectively. With the adopted step of 0.02 in the parameters of the gamma, Lorentzian and Gaussian functions (Eqs. (10)-(12)), this resulted in 15 records, and Table 2 presents 5 representative cases with the highest rates of SP-IP occurrence. We believe that case (1) is the most plausible one and Figs. 1-3 present a visual comparison of the observed and simulated distribution corresponding to this case ${ }^{3}$. Below we discuss some aspects of our analysis, which are not reflected in Table 2.

\subsubsection{Pulsar period}

Both gamma (Eq. (10)) and Lorentzian (Eq. (11)) functions with parameter values $m=0.34 \pm 0.02, a=2.52 \pm 0.04$ and $x_{0}=0.62 \pm 0.02, a_{0}=0.4 \pm 0.02$ respectively, are plausible parent density distribution functions. However, the gamma function seems much better suited to reflect the skewed character of the pulsar period distribution. The Gaussian function (Eq. (12)) is rather unlikely. In the best case corresponding to $x_{0}=0.73$ and $\sigma_{0}=0.36$, the significance $\mathcal{P}$ is only about 0.0003 .

\subsubsection{Inclination angle}

ZJM03 argued, ignoring the issue of the interpulse emission, that the parent distribution of the magnetic inclination angles

\footnotetext{
${ }^{3}$ Figures 1-3 compare the distributions in a linear scale. Given the many orders of magnitude covered by the period and pulse width, it is worthwhile to compare distributions in a log-scale. We have binned the logarithm of the period pulse width and found that the results both for histograms and K-S tests do not change.
} 
can be expressed by the cosine function or modified cosine function represented by Eq. (9) in this paper. Including the analysis of interpulse statistics we confirm the latter but refute the former, since the cosine function generates less than $0.3 \%$ of DP-IP (as compared with about $2 \%$ observed). This is a strong conclusion since better statistics of interpulse occurrences will not alter it. The sine-like function (Eq. (7)) generates too many DP-IP $(\sim 5 \%)$ and far too few SP-IP $(\sim 0.02 \%)$, while the interpulse rates generated by the flat distribution function (Eq. (6)) are roughly comparable with observations, especially when associated with the meridionally compressed beam (Eq. (17)), but the results of the K-S test for the magnetic inclination angles are not promising $\left(\mathcal{P}<10^{-5}\right)$. Therefore, the modified cosine function (Eq. (9)) is the only plausible density distribution function that satisfies all constraints. We have checked whether one can improve the statistical results by changing values of the parameters in Eq. (9). It appeared that without violating the basic condition that $\mathcal{P}>0.001$ for all considered quantities $\left(P, W_{10}\right.$ and $\left.\alpha\right)$, one can only increase the interpulse rates by a small fraction (e.g. from $0.74 \%$ to $0.77 \%$ for SP-IP).

\subsubsection{Beaming fraction}

For the 15 cases satisfying all constraints adopted in our analysis, the beaming fraction $f$ defined as the number of detected pulsars divided by 50000 detection attempts is $0.124 \pm 0.004$. This is consistent with the probabilities of observing a normal radio pulsar obtained by both TM98 and ZJM03. One should emphasize that this beaming fraction is only a mean value, which is biased by the underlying period distribution. The actual beaming fraction for a given pulsar is obviously perioddependent, as can be seen from Eqs. (2)-(4).

\section{Discussion and conclusions}

In this paper we performed statistical studies using the Monte Carlo simulations of the possible parent distributions of pulsar periods $P$ and magnetic inclination angles $\alpha$. We generated synthetic distributions of the pulse-widths $W_{10}$, as well as the interpulse occurrences, and compared them with the observational data. We found that the observed distributions of pulsar periods, pulse widths and inclination angles are relatively easy to reproduce with a variety of trial density distribution functions. However, when we used the criterion that the $\mathrm{K}-\mathrm{S}$ significance probabilities $\mathcal{P}$ for $P, W_{10}$ and $\alpha$ are higher than 0.001 and that the generated interpulse rates agree with the observed rates, we were left with just a few possibilities, presented in Table 2. Our results can be summarized as follows:

(1) The distribution of the magnetic inclination angles in the parent pulsar population is a complicated function represented by Eq. (9), which has a local maximum around $\alpha=25^{\circ}$ and another weaker one around $\alpha=90^{\circ}$. This function reproduces the observed distribution of the observed inclination angles (Fig. 3), as well as generates the rates of occurrence of interpulses at observed levels.
(2) The parent distribution of periods in the normal pulsar population (excluding millisecond and other recycled pulsars) can be described by the gamma function (Eq. (10)) with parameters $m=0.34 \pm 0.02$ and $a=2.52 \pm 0.04$. The actual values of these parameters differ slightly depending on the $\rho$-P relation (Eqs. (2)-(4)).

(3) There is no evidence that the shape of the pulsar beam deviates significantly from a circular crossection. It seems that a binomial distribution of the opening angles represented by Eq. (4) is the preferred one (especially the version referred as to Eq. (4a)).

(4) The beaming fraction $f$, that is the mean value of the fraction of observable pulsars or the mean probability of observing a normal pulsar, is $0.124 \pm 0.004$.

In general, our results are consistent with those of ZJM03. However, we added the constraints related to the interpulse analysis and found that this aspect of our analysis is the most restrictive one. We were able to reject the pure cosine distribution of the inclination angles, but we confirmed their modified cosine function which has a local maximum around $\alpha=25^{\circ}$ and another weaker one near $\alpha=90^{\circ}$. It seems that this function should be treated as a model rather than the true underlying distribution, as it is hard to justify physically why the distribution should have peaks near $25^{\circ}$ and $90^{\circ}$ (which effectively means a deficit of the inclination angles at small values and an excess at large values with respect to a pure cosine distribution function). Nevertheless, this means that the evolution of the magnetic inclination angles in pulsars cannot be described by any simple law (alignment, counter-alignment, etc.). However, the competition between the alignment (due to magnetic dipole radiation) and counter-alignment (due to magnetospheric currents associated with pulsar wind) could perhaps lead to such a complicated underlying distribution function.

Although we improved the analysis of ZJM03 by adding the interpulse statistics, we are still lacking an analysis of the possible effect of the intrinsic luminosity of radio pulsars on our results. This problem is, however, very difficult and complicated and we will postpone a full treatment of this to a subsequent paper. The proper approach would be to compare the synthetic radio luminosity with the minimum detectable flux achieved in a given pulsar survey, and thus it can be applied only to uniform data sets of pulsars detected in single survey. Our data do not have such a degree of uniformity. However, most surveys were less sensitive to long-period pulsars, as it follows from the nature of the applied Fourier-transform method. Since the interpulse emission (which appears to be the most restrictive constraint in our analysis) occurs mainly at shorter periods, a possible under-representation of pulsars with longer periods should not significantly affect our general results.

Acknowledgements. This work is partially supported by the Grant 1 P03D 02926 of the Polish State Committee for Scientific Research. We thank E. Gil and U. Maciejewska for technical help. We also thank the anonymous referee for very constructive and helpful criticism. 


\section{References}

Backer, D. C. 1976, ApJ, 209, 895

Biggs, J. D. 1990, MNRAS, 245, 514

Gil, J. 1981, Acta Phys. Pol., B12, 1081

Gil, J. 1983, A\&A, 127, 267

Gil, J. A., \& Han, J. L. 1996, ApJ, 458, 265 (GH96)

Gil, J., Gronkowski, P., \& Rudnicki, W. 1984, A\&A, 132, 312

Gil, J., Kijak, J., \& Seiradakis, J. H. 1993, A\&A, 272, 268 (GKS93)

Gould, D. M. 1994, Ph.D. Thesis, University of Manchester

Gould, D. M., \& Lyne, A. G. 1998, MNRAS, 301, 235

Graham-Smith, F. 2003, The radio emission from pulsars, Reports on Progress in Physics, 66, 173

Hankins, T. H., \& Fowler, L. A. 1986, ApJ, 304, 256

Henry, G. R., \& Paik, H. J. 1969, Nature, 224, 1188

Kramer, M., Wielebinski, R., Jessner, A., Gil, J. A., \& Seiradakis, J. H. 1994, A\&AS, 107, 515

Kramer, M., Bell, F. J., Manchester, R. N., et al. 1994, MNRAS, 342, 1299

Lyne, A. G., \& Manchester, R. N. 1988, MNRAS, 234, 477

Manchester, R. N., \& Lyne, A. G. 1977, MNRAS, 181, 761

Manchester, R. N. 1978, Proc. Astr. Soc. Aust., 3, 200
Manchester, R. N. 1996, in Pulsars: Problems and Progress, ed. S. Johnston, M. A. Walker, \& M. Bailes (San Francisco: Astronomical Society of the Pacific), IAU Coll., 160, 193

Manchester, R. N., \& Lyne, A. G., Camilo, F., et al. 2001, MNRAS, 328, 17

McKinnon, M. M. 1993, ApJ, 413, 317

Mitra, D., \& Deshpande, A. A. 1999, A\&A, 346, 906

Morris, D. J., Hobbs, G., Lyne, A. G., et al. 2002, MNRAS, 335, 275

Press, W. H., Teukolsky, S. A., Vatterling, W. T., \& Flannery, B. P. 1992, Numerical Receipes (Fortran) (Cambridge University Press)

Prószyński, M. 1979, A\&A, 79, 8

Rankin, J. M. 1990, ApJ, 352, 247

Rankin J. M., 1993a, ApJ, 405, 285

Rankin J. M., 1993b, ApJS, 85, 145

Rickett, B. J., \& Lyne, A. G. 1968, Nature 218, 934

Roberts, D. H., \& Sturrock, P. A. 1972, ApJ, 172, 435

Roberts, D. H., \& Sturrock, P. A. 1973, ApJ, 181, 161

Taylor J. H., Manchester R. N., \& Lyne A. G., 1993, ApJS, 88, 529 (Pulsar Catalog)

Tauris, T. M., \& Manchester, R. N. 1998, MNRAS, 298, 625 (TM98)

Zhang, L., Jiang, Z.-J., \& Mei, D.-Ch. 2003, PASJ, 55, 461 (ZJM03) 